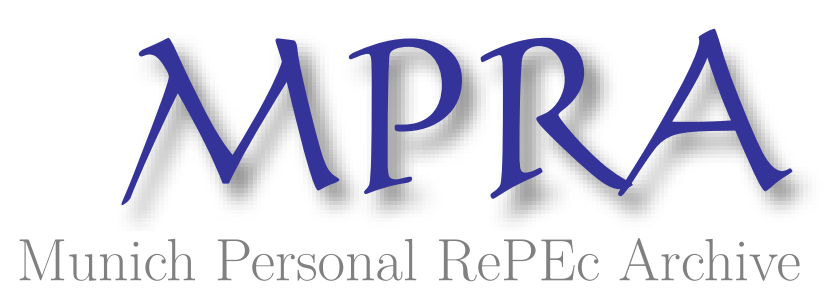

\title{
Social choice and the closed convergence topology
}

Chichilnisky, Graciela

12 July 1990

Online at https://mpra.ub.uni-muenchen.de/8353/

MPRA Paper No. 8353, posted 21 Apr 2008 03:04 UTC 


\title{
Social choice and the closed convergence topology
}

\section{Graciela Chichilnisky*}

Department of Economics, Columbia University. New York, NY 10027, USA

Rcocived July 12, 1990/Accepted January 23, 1991

\begin{abstract}
This paper revisits the aggregation theorem of Chichilnisky (1980), replacing the original smooth topology by the closed convergence topology and responding to several comments ( $\mathrm{N}$. Baigent $(1984,1985,1987,1989)$, N. Baigent and P. Huang (1990) and M. LeBreton and J. Uriarte (1990a, b). Theorems 1 and 2 establish the contractibility of three spaces of preferences: the space of strictly quasiconcave preferences $P_{\text {SCO }}$, its subspace of smooth preferences $P_{\text {Sco }}^{\text {s }}$, and a space $P_{1}$ of smooth (not necessarily convex) preferences with a unique interior critical point (a maximum). The results are proven using both the closed convergence topology and the smooth topology. Because of their contractibility, these spaces satisfy the necessary and sufficient conditions of Chichilnisky and Heal (1983) for aggregation rules satisfying my axioms, which are valid in all topologies. Theorem 4 constructs a family of aggregation rules satisfying my axioms for these three spaces. What these spaces have in common is a unique maximum (or peak). This rather special property makes them contractible, and thus amenable to aggregation. However, these aggregation rules cannot be extended to the whole space of preferences $\boldsymbol{P}$ which is not contractible and therefore does not admit continuous aggregation rules satisfying anonymity and unanimity, Chichilnisky $(1980,1982)$. The results presented here clarify an erroneous example in LeBreton and Uriarte (1990a, b) and respond to Baigent $(1984,1985,1987)$ and Baigent and Huang (1990) on the relative advantages of continuous and discrete approaches to Social Choice.
\end{abstract}

\section{Introduction}

Recent papers by Baigent and Huang (1990) and Baigent (1984, 1985, 1987), LeBreton and Uriarte (1990a, b), make interesting comments on some of my theorems in Social choice and on other results following my approach. Baigent and

* Comments from Geoffrey M. Heal, Andreu Mas Colell, Jcan Francois Mertens and Maurice Salles are gratefully acknowledged. Research support was provided by NSF SES 8409857. 
Huang (1990) and Baigent $(1985,1987)$ reinterprct my results in a discrete framework, and argue in favor of the discrete approach as preferable to the formulation of Social Choice problems in a continuous fashion. I argue here that both approaches are useful, but the continuous approach scems necessary to obtain certain fundamental results, such as necessary and sufficient conditions for resolving the social choice paradox (Chichilnisky and Heal 1983), results which could not be obtained in a discrete framework. In addition, a continuous approach allows the use of calculus and topology and thus helps bridge the gap between social choice and the theory of markets, e.g. Chichilnisky (1990 b). This cannot be achieved with the discrete approach. LeBreton and Uriarte (1990a, b) argue in favour my topological approach but attempt to test the robustness of my results by changing the topology to the closed convergence topology and claiming to construct a counterexample. This they attempt to do by constructing an aggregation map which satisfies my axioms on a space of strictly quasiconcave preferences denoted $\boldsymbol{P}_{\mathrm{SCO}}$, a space which they claim "is identified with" a sphere (page 5, line 8), while my theorem proves that spheres do not admit such aggrcgation maps (Chichilnisky 1982). Their argument is flawed because of a topological miscalculation: their claim that $P_{\mathrm{SCO}}$ is a sphere is incorrect. Theorem I establishes that $P_{\mathrm{Sco}}$ is a contractible space, both in the closed convergence and (its smooth version $P_{\mathrm{s}}^{S}$ ) in the smooth topologies. Because of its contractibility, $P_{\text {Sco }}$ cannot be identified with a sphere. Contractibility is also why $P_{\text {sco }}$ admits aggregation maps respecting my axioms: this is the result proved in Chichilnisky and Heal (1983). Their example is therefore a corollary of the Chichilnisky-Heal 1983 theorem, thus showing the robustness of our results when the topology is changed and the closed convergence topology is adopted. Theorems 1 and 2 establish the contractibility of $P_{\mathrm{SCO}}$ by constructing a deformation retract of $P_{\mathrm{SCO}}$ into a space of one-peaked preferences which is, in turn, homeomorphic to the choice space $X$. Since the choice space $X$ is convex, $P_{\mathrm{SCO}}$ is contractible, and definitely not a sphere. Theorem 2 establishes the same result for the space of smooth preferences in $P_{\mathrm{Sco}}$, denoted $P_{\mathrm{Sc}}^{S}$, and also for a space $P_{1}$ of non convex smooth preferences with a unique maximum. All these spaces have one thing in common: they have a unique interior critical point, a maximum, on $X$ (also called one peak). This special property makes them contractible, and amenable to social aggregation'. Theorem 4 constructs (always within the space of one peaked preferences) a large family of convex-type aggregation map respecting unanimity and anonymity and continuous under both topologies, for $P_{\mathrm{SCO}}, P_{\mathrm{SCO}}^{S}$ and $P_{\mathrm{L}}$. These maps are different from the maps proposed by LeBreton and Uriarte, because the latter are not well defined on smooth preferences. The existence of such convex-like aggregation maps exhibits clearly the special structure of the space of preferences $\boldsymbol{P}_{\mathrm{sco}}$, and explains its restricted nature. In contractible spaces there is no difficulty in finding appropriate aggregation maps: this is the result in Chichilnisky and $\mathrm{Heal}$ (1983), which is true for any topology. The problem emerges when trying to construct appropriate aggregation maps for the whole space of preferences, which is not contractible. The space of all preferences does not admit aggregation maps satisfying my axioms (Chichilnisky 1980), nor do LeBreton and Uriarte attempt to construct one. Therefore, their work does not really deal with the robustness of my impossibility results; it simply shows that

Indeed, the spacc of smooth preferences with either a unique maximum or a unique minimum on $X$, is not contractible. 
we can aggregate preferences over restricted domains. This is of course well known since the work of Black in 1948, and the necessary and sufficient conditions of Chichilnisky and Heal (1983). No new information is provided by constructing aggregation rules for spaces of contractible preferences.

\section{Robustness of the impossihility theorem}

Definitions. Let the choice space $X$ be a closed convex subset on $R^{n}$. The space $P_{\text {sco }}$ is defined as the space of strictly quasiconcave continuous preferences on $X$ with the closed convergence topology. $P_{\mathrm{SCO}}^{S}$ is the space of smooth preferences in $P_{\text {sco }}$. where a smooth preference is one which is represented by a $C^{2}$ real valued function defined on a neighborhood of $X$. The space of smooth preferences $P_{1}$ is defined as those smooth preferences $p$ with a single interior critical point $p_{m}$ in $X$, a maximum, and which are regular under the radial coordinate system of $X-p_{m}{ }^{2}$. All these spaces of preferences can be described as "onc peaked" in the sense that they have a single local maximum, and their minima are necessarily in the boundary of $X$.

Note that $P_{\mathrm{sco}}$, which is the space used in LeBreton and Uriarte (1990a) is an incomplete space: many Cauchy sequences in $P_{\mathrm{sco}}$ converge to points outside of $P_{\mathrm{Sco}}$. For example, linear preferences are the limit of preferences in $P_{\mathrm{sco}}$ but are not contained in $P_{\text {sco }}$. Similarly quasiconcave (rather than strictly quasi concave) preferences are the limit of strictly quasi concave preferences but are not contained in the space $P_{\mathrm{SCO}}$. The aggregation rule proposed by Le Breton and Uriarte does not extend to quasiconcave preferences, nor to spaces with multiple-peaked preferences. An analogy with the single peaked profiles of preferences may prove useful here. It has been known since the work of Black in 1948 , that a solution to the aggregation problem can be found within the space of single peaked profiles. The problem has always been that the aggregation rules which one obtains for such preferences do not extend to larger classes of preferences. As soon as the class of profiles is enlarged the proof, which relies crucially in singlepeakedness, nor longer holds, and the existence theorem is lost. In particular, the spaces of all continuous or smooth preferences which have proven so useful to analyse properties of markets and of spaces of preferences, are excluded from this approach.

A similar problem arises from LeBreton and Uriarte's result. They find a continuous, anonymous aggregation rule satisfying unanimity, but only for a restrictive space of preferences $P_{\mathrm{SCO}}$. Their construction does not generalize to the space of all continuous preferences. As already mentioned the space $P_{5 c 0}$ is, furthermore, incomplete, in the sense that Cauchy sequences of preferences in $P_{\text {SCO }}$ have limits outside $P_{\text {SCO }}$. Continuity properties in such an incomplete space are of limited interest. Continuity of a function $f$ requires that if a sequence $\left(x_{n}\right) \rightarrow x$, then $f\left(x_{n}\right) \rightarrow f(x)$. When the condition that $\left(x_{n}\right) \rightarrow x$ is not satisfied, because of incompleteness, then the continuity requirement is empty. Continuity

2 This is the standard radial coordinate system in $R^{n}$ with the orgin shifted to $p_{m}$. Regularity of a $C^{2}$ function $f: X \rightarrow R$ in this coordinate system implies that its derivative $D f$ on $X-p_{m}$ with these coordinates does not vanish, and in particular an indifference surface of $f$ intersects each line $t$ through $p_{m}$ at one point.

${ }^{3}$ Note that profiles of "one peaked preferences" need not be "single-peaked profiles" as definod usually in the social choice literature. 
"looses its bite". It is much easier to obtain continuity, because it is more difficult to obtain convergent sequences in an incomplete space. The whole meaning of continuity in such a space is questionable.

$P_{\text {Sco }}$ is also topologically trivial or contractible. Our next step is to establish the topological structure and in particular the contractibility, of $P_{\mathrm{SCO}}$. Recalling Chichilnisky and Heal (1983) show that the topological complexity of the space of all preferences obstructs the construction of social choice rules, it comes as no surprise that the problem of appropriate aggregation does not exist in this topologically trivial space.

Theorem 1. The space of strictly quasiconcave preferences $P_{\mathrm{sco}}$ on a compact convex choice set $X \subset R^{n}$ with the closed contergence topology is contractible.

Proof. The proof consists of showing that $P_{\mathrm{sco}}$ admits a deformation retract ${ }^{4}$ onto a space of preferences which is in turn homeomorphic to the space of choices $X . X$ is a convex set and in particular contractible. Therefore $P_{\mathrm{sco}}$ is also contractible. The deformation retract $H$ continuously deforms the space $P_{\text {sco }}$ into the space $S$ of "standard" one-peaked preferences, which are defined as those one-peaked preferences having as indifference surfaces concentric spheres around the single peak (intersected with the choice space $X$ ). By mapping each preference to one which is the "standard" preference with the same peak, we obtain a deformation retract of $P_{\mathrm{sco}}$ into the space $S$ of standard one-peaked preferences on $X$, which is clearly homeomorphic to $X$ itself. $P_{\text {Sco }}$ is thus contractible.

The first step is to represent a preference in $P_{\text {sco }}$ by a couple consisting of its peak and a family of functions difining its concave indifference surfaces. Formally, let $U: P_{\mathrm{SCO}} \rightarrow U_{\mathrm{SCO}}$ be the continuous utility representation for $P_{\mathrm{SCO}}$ which exists by Theorem 1 , page 137 , of LeBreton and Uriarte $(1990 \mathrm{a}) . U(p)$ is also denoted $u_{p}$. Without loss of generality we may assume that all utilities in $U_{\text {sco }}$ assume the minimum valuc 0 and the maximum valuc 1 on the compact set $X$. By the mean value theorem they will assume all values in the interval $[0,1]$. Now assign to any $p$ in $P_{5 c 0}$ a pair $(x, f)$, where $x \in X$ is the peak of $p$, and $f$ is a continuous function from the unit interval $[0,1]$ into $C\left(S^{n-1}, R^{n}\right)$, the space of continuous functions from $S^{n}{ }^{1}$ to $R^{n}$ endowed with the sup norm. For each $t, f(t)$, also denoted $f_{t}$, is a continuous function from the unit sphere $S^{n-1}$ to $R^{n}$ so that $f:[0,1] \rightarrow C\left(S^{n-1}, R^{n}\right)$ is a continuous path of such functions. For each $t$ in $[0,1]$, define $f_{i} \in C\left(S^{n-1}, R^{n}\right)$ by:

$$
f(\alpha)=R_{a, \alpha} \cap \operatorname{Bdry}\left\{y \in X: u_{p}(y)>t\right\}^{5},
$$

where $u_{p}=U(p)$, and where $R_{\alpha, x}$ is defined as the ray with origin $x$ in the direction $\alpha$ if $x \in \dot{X}^{6}$, and as its projection on Bdry $X$ if $x \in \operatorname{Bdry} X$; when $R_{\alpha, x}=\{x\}$, then $f_{i}(\alpha)=x$, see Fig. 1 .

\footnotetext{
4 A retraction $r: X \rightarrow A \subset X$ is a continuous map such that when restricted to $A$, it is the identity map, i.e. $r / A=$ id: $A \rightarrow A$. A subset $A$ of a space $X$ is a deformation retract of $X$ if there exist a retraction map $r: X \rightarrow A$ and a homotopy $f: X \times[0,1] \rightarrow X$ such that for all $x \in X, f(x, 0)=x$, $f(x, 1)=r(x)$, and $f(a, t)=a$ for all $a \in A$, and all $t \in[0,1]$. If $A$ is a deformation retract of $X$, then for all $n$, the inclusion map $i: A \rightarrow X$ induces an isomorphism of the $n$-th homotopy groups $A$ and of $X$, see Spanier (1966), Theorem 13, Chapter 1.4.

Bdry $(Y)$ denotes the boundary of the set $Y$.

$6 \dot{X}$ denotes the interior of the set $X$.
} 

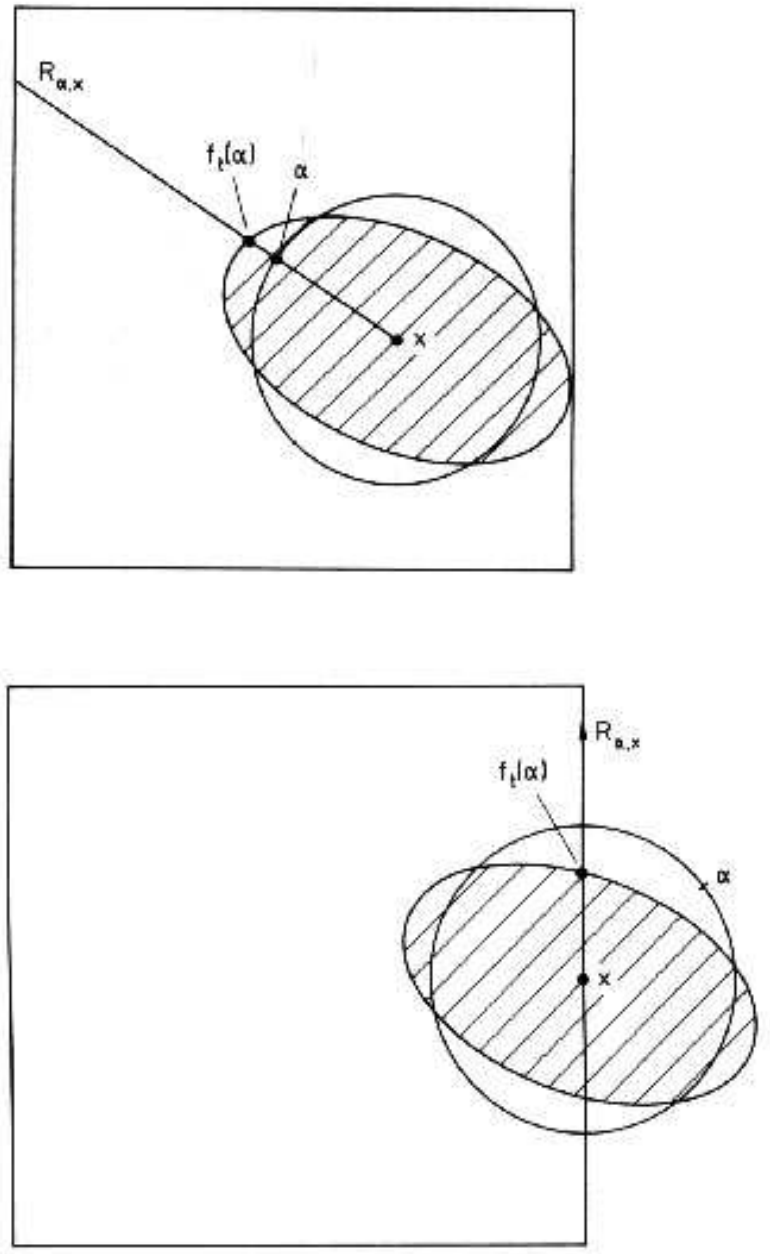

Fig. 1. The shaded area represents the set $\left\{y \in X: u_{p}(y)>t\right\}$

Let $F=\left\{\varphi: \varphi:[0,1] \rightarrow C\left(S^{n-1}, R^{n}\right), \varphi\right.$ continuous $\}$, and endow $X \times F$ with the product topology. The procedure constructed above defines a map $\Gamma: P_{\mathrm{SCO}} \rightarrow X \times F$ which is continuous by the strict quasiconcavity of the preferences in $P_{\mathrm{sco}} . \Gamma$ is a representation of preferences by peaks and by families of functions giving their indifference surfaces. For each peak and family of functions $(x, f)$ in $\Gamma\left(P_{\mathrm{sco}}\right)$, we can construct a preference $p$ mapping into it, i.e. such that $\Gamma(p)=(x, f)$. This defines a map $\Pi$ from $\Gamma(X \times F)$ back into $P_{\text {sco }}$ which is a left inverse of $\Gamma$. Formally, for any $(x, f) \in \Gamma\left(P_{\mathrm{Sco}}\right)$, let $\Pi(x, f)$ be the preference $p$ with the (single) peak $x$ and such that for all $y \in X$, the set of points $p_{v}$ preferred to $y$ in $X$ is the convex set bounded by the image in $X$ of the sphere $S^{n-1}$ under $f_{t}$, for $t=u_{p}(y), \Gamma\left(u_{p}\right)=u_{p} \in U_{\mathrm{SCO}}$. In other words, $\Pi$ maps any $(x, f)$ in the image of $P_{\text {sco }}$ into the preference with (a single) peak at $x$ and having as indifference surfaces $\left\{f_{t}\left(S^{n-1}\right)\right\}, t \in[0,1]$. The map $I$ is clearly continuous on the $\Gamma\left(P_{\text {SCO }}\right)$. The composition of the maps $\Gamma$ and $\Pi$, indicated by the diagram 


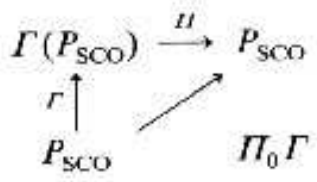

and denoted $\Psi=\Pi \circ \Gamma: P_{\mathrm{SCO}} \rightarrow P_{\mathrm{SCO}}$ is continuous under the closed convergence topology on $P_{\mathrm{SCO}}$, and it maps any preference $p$ in $P_{\mathrm{Sco}}$ into itself. The next step is to define a deformation retract $H$ from $P_{\mathrm{sco}}$ onto the space of "standard" preferences $S$. This is achieved in several steps. First define the map $T: X \times F \rightarrow \Gamma(S) \subset \mathrm{X} \times \mathrm{F}$ to be the continuous map $T(x, f)=(x$, id), where id: $S^{n-1} \times[0,1] \rightarrow R^{n}$ is such that for all $t \in[0,1]$

$$
\begin{aligned}
& \mathrm{id}_{l}: S^{n-1} \rightarrow \operatorname{Bdry}\left(B_{i}^{n} \cap X\right), \\
& \text { id, }(\alpha)=R_{a, x} \cap \operatorname{Bdry}\left(B_{i}^{n} \cap X\right),
\end{aligned}
$$

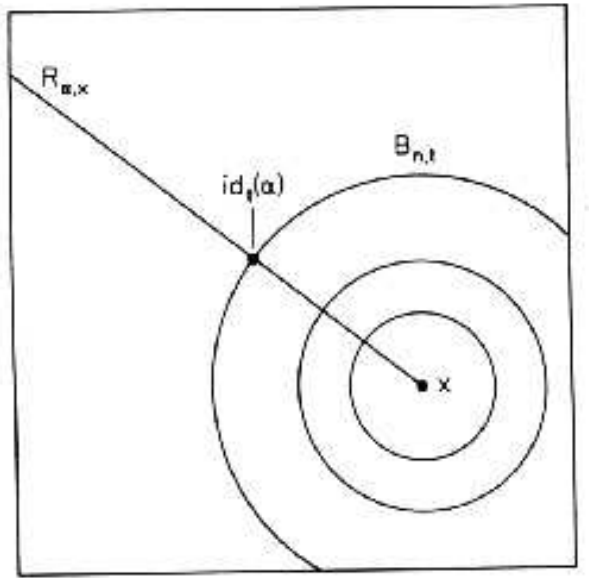

Fig. 2. The map id, $: S^{n}{ }^{1} \rightarrow \operatorname{Bdry}\left(B_{i}^{n} \cap X\right)$. Note that $\mathrm{id}_{0}(\alpha)=x$ for all $\alpha$ in $S^{n-1}$, i.e. id maps $S^{n-1}$ into $\{x\}$

where $B_{i}^{n}$ is the $n$ dimensional ball of radius $\sigma . t$ centered at $x$, and $B_{1}^{n}$ is a ball of radius $\sigma$, the smallest ball centered in $x$ which covers $X$, sec Fig. 2 . Next we define a retraction $R$ which maps $P_{\mathrm{SCO}}$ into its subspace $S$ consisting of standard preferences, by the diagram

$$
P_{\mathrm{sco}} \stackrel{r}{\longrightarrow} \Gamma\left(P_{\mathrm{sCO}}\right) \stackrel{T}{\longrightarrow} \Gamma(S) \stackrel{\Pi}{\longrightarrow} S
$$

i.e. $R(p)=\Pi \circ T \circ \Gamma(p)$. Then $R: P_{\mathrm{SCO}} \rightarrow S$ and $R(p)=p$ for all $p \in S$. The retraction $R$ assigns to each preference in $P_{\mathrm{Sco}}$ the "standard" preference with the same peak, and $S$ is invariant under $R$.

Finally we construct a deformation retract of $P_{\text {sco }}$ onto $S$, i.e. an homotopy $H: P_{\mathrm{SCO}} \times[0,1] \rightarrow P_{\mathrm{SCO}}$ which is the identity map on $P_{\mathrm{SCO}}$ when $\lambda=0$, and the retraction $R$ onto $S$ when $\lambda=1$. This map consists of representing a preferences (via $\Gamma$ ) in the space $X \times F$, then gradually deforming within $X \times F$ the indifference surfaces of each preference until they became spheres, and then projecting the 
result (by $\Pi$ ) back onto the subset $S$ of $P_{\mathrm{sco}}$. Formally, for each $\lambda \in[0,1]$ and $p \in P_{\mathrm{SCO}}$ :

$$
H(p, \lambda)=\Pi \circ \Omega_{\lambda} \circ \Gamma(p),
$$

where

$$
\Omega_{\lambda}(x, f)=(1-\lambda) f+\lambda \text { id },
$$

and

$$
(x, f)=\Gamma(p),
$$

and where $\lambda f$ is defined by $(\lambda f)(t)=\lambda f(t)$ for all $t \in[0,1]$. In other words,

$$
\begin{aligned}
& \mathrm{H}(p, \lambda)=\Pi(x,(1-\lambda) f+\lambda \text { id }) \in P_{\mathrm{sco}}, \\
& \Gamma(p)=(x, f) .
\end{aligned}
$$

Then for all $p$ in $P_{\mathrm{sco}}$, and all $x \in[0,1], H$ is continuous and it satisfies:

$$
\begin{aligned}
& H(p, 0)=\Pi(x, f)=p \\
& H(p, 1)=\Pi(x, \text { id })=R(p) \in S
\end{aligned}
$$

and

$$
H(p, t)=p \text { for all } p \in S \text {. }
$$

Therefore $I$ defines a deformation retract of $P_{\text {sco }}$ onto $S \subset P_{\text {sco }}$. The space $S$ is clearly homeomorphic to the choice space $X$. Since $X$ is convex, $X$ is contractible. Therefore so is $S$. Since $P_{\text {sco }}$ has $S$ as a deformation retract, it follows that $P_{\text {sco }}$ is contractible too (Spanier 1966).

Observation. We have shown that the space $P_{5 \mathrm{SCO}}$ is contractible with the closed convergence topology on preferences. Therefore the statement made by LeBreton and Uriarte that the space $P_{\mathrm{SCO}}$ is "identified with" $S^{n-1} \cup\{0\}$ (Page 5, line 8), is not correct, since $S^{n-1} \cup\{0\}$ is not contractible. It is possible that what these authors meant, instead, is that the space $P_{\text {sco }}$ is equivalent to $S^{n-1} \cup\{0\}$ in the smooth topology that 1 utilize, since they use the words "locally equivalent to $S^{n-1} \cup\{0\}^{\prime}$. However, even with this interpretation their statement is incorrect. Their space $P_{\mathrm{SCO}}$ consists of one-peaked preferences which may not have a well defined gradients at each point, while the topology which I utilize, which coincides with one used in Debreu (1972), requires well defined gradients for its definition. Typically the preferences in $P_{\text {sco }}$ have "kinks" in their preferred sets, for otherwise the operation of taking the minimum of two strictly quasiconcave functions, which is the aggregation map defined by LeBreton and Uriarte, would not map into their space $P_{\text {soo }}$. These kinks prevent the definition of a "most preferred direction" (i.e. gradients) and therefore makes it impossible to define the smooth topology, which is the topology commonly used on smooth preferences. In sum: $P_{\text {SCo }}$ cannot be a sphere with the smooth topology since the smooth topology is not even defined on $P_{\mathrm{sco}}$. Therefore the statement that the space $P_{\mathrm{SCO}}$ is equivalent to a sphere plus a point, in either the closed convergence topology which they use or in the smooth topology which I utilize, is incorrect. In an effort to clarify the matter I consider yet a third possible interpretation of their statement: 
that the space of smooth preferences in $P_{\mathrm{SCO}}$, denoted $P_{\mathrm{SC},}^{S}$, could be a sphere. Yet this is also incorrect as the following result shows:

Theorem 2. The space $P_{1}$ of smooth one-peaked preferences on the convex choice $X$ with the smooth topology is contractible, and so is its subspace $P_{\mathrm{Sco}}^{s}$ of strictly quasi concave smooth preferences.

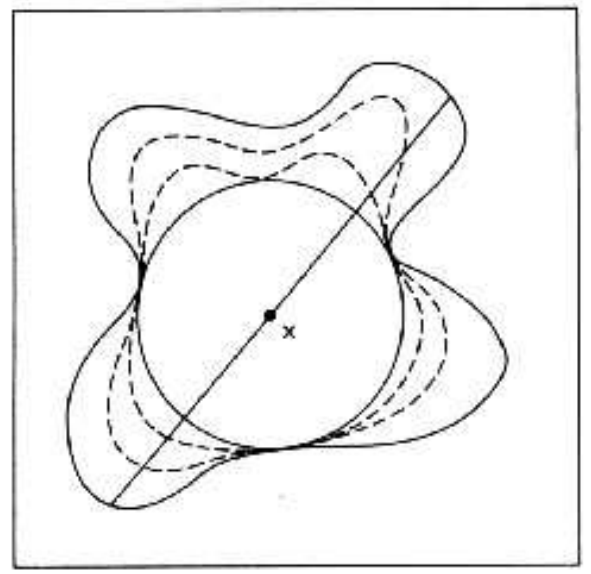

Fig. 3. Deforming a non-convex smooth preference in $P_{1}$

Proof. This follows from the proof of Lemma 1, observing that the deformation retract $H$ in Theorem $I$ is also a continuous map under the smooth topology of Chichilnisky (1980), when restricted to the spaces $P_{\mathrm{SCO}}^{s}$ and $P_{1}$, see Fig. 3.

The necessary and sufficient theorem of Chichilnisky and Heal (1983) proves the existence of a continuous anonymous aggregation rule for any number of voters, if and only if the space of preferences is contractible. As a corollary of Theorem 2 and of the 1983 theorem we have thus obtained:

Theorem 3. The spaces of preferences $P_{\mathrm{SCO}}, P_{\mathrm{Sco}}^{5}$ and $P_{1}$ all admit continuous, anonymous aggregation rules respecting unanimity for any number of agents.

Proof. This is a corollary from the necessary and sufficient conditions in Chichilnisky and Heal (1983).

The paper by LeBreton and Uriarte exhibits one continuous map satisfying unanimity and anonymity on $P_{\mathrm{sco}}$. However, as already noted their map is not well defined on $P_{\mathrm{ScO}}^{S}$ because it consists of taking the minima of two maps, and in general smoothness is lost. It is of interest to construct examples of well defined maps which map profiles of smooth preferences into smooth preferences satisfying the desired axioms. The following Theorem constructs a family of such maps for spaces of smooth preferences including preferences which are not necessarily convex. 
Theorem 4. For any $k>0$, and any positive vector $\lambda=\left(\lambda_{1}, \ldots, \lambda_{k}\right) \in R^{k}$ satisfying $\Sigma \lambda_{i}=1$, let $\phi_{\lambda}:\left(P_{\mathrm{SCO}}\right)^{k} \rightarrow P_{\mathrm{SCO}}$ be defined by $\phi_{\lambda}\left(p_{1}, \ldots, p_{k}\right)=\Sigma_{\lambda} \circ \Gamma(p)$, where $\Gamma$ is as in Theorem 1, and $\Sigma_{\lambda}\left[\left(x_{1}, f^{1}\right), \ldots\left(x_{k}, f^{k}\right)\right]=\left(\Sigma \lambda_{i} x_{i}, \Sigma \lambda_{i} f^{\prime}\right)^{7}$. For any $k>0$, and each $\lambda$ define similarly $\phi_{\lambda}^{1}:\left(P_{1}^{k}\right) \rightarrow P_{1}$ as $\Sigma_{\lambda} \circ \Gamma$. Then $\phi_{\lambda}$ and $\phi_{\lambda}^{1}$ are well defined, anonymous and respect unanimity. $\phi_{\lambda}$ is continuous with the closed convergence topology, and $\phi_{\lambda}^{1}$ is continuous with the smooth topology.

Proof, This follows from the properties of $\Gamma$ and of $\Sigma_{\lambda}$.

A last comment on LeBreton and Uriarte will clarify their use of the words "local" and "global" when referring to my and their approach respectively. Their paper does not give a reason for this notation, which could be inadvertedly misleading. Their notation of "local" for my approach suggests for example that the gradient of the social preference at one point may depend solely on the gradients of the individual preferences at the same point. This is not correct. Acceptable aggregation rules in my approach may assign two different gradients at one choice $x$ to different profiles of preferences, even when both profiles have the same gradients at $x$, provided that at another choice $y$ the gradients are not the same. This shows that acceptable aggregation rules take into account the gradients of the profiles everywhere (globally) and not just at a single point, when finding the gradient of the social preference at that point. My approach is global rather than local. It is also clear that the topology I use, which coincides with one utilized in Debreu (1972) when studying smooth preferences in the context of general equilibrium theory, requires global proximity of gradients. It requires that preferred directions be close at all choices, for the preferences to be close. In other words, the word "local" to describe my approach or my topology (or the acceptable social choice rules) does not seem appropriate.

\section{Baigent and Huang}

In three interesting articles, Baigent $(1984,1985,1987)$ proves discrete versions of the impossibility theorem of Chichilnisky $(1980,1982)$ which, inter-alia, throw light on the role of continuity and show that continuous spaces of choices are not essential to prove results similar to my 1980 results. In Baigent and Huang (1990) the authors go a step further. On the strength of the Baigent results they argue that the continuous framework may not be necessary, and that discrete techniques always suffice. They agree with my results and my topology but not with the general approach. They favour the more traditional discrete approach to the continuous approach.

My response to Baigent and Huang is, in brief, that we need a continuous approach because we need calculus and topology in Social Choice theory. There are two practical reasons. One is to obtain new and useful results which are not obtainable, not even understandable, otherwise. Examples are the necessary and sufficient conditions mentioned above to resolve the paradox, the topological correspondence of the Social Choice paradox with fixed point theorems, which are at the core of the existence of equilibria, and the game theoretical approaches about manipulation of games, all obtained with this approach in recent years (see e.g. Chichilnisky 1979, 1980, 1982, and Chichilnisky and Heal 1983). A

\footnotetext{
The sum $\Sigma \lambda_{i} f^{\prime}$, where $f^{i}: S^{n-1} \rightarrow R^{n}$ is defined by $\left(\Sigma \lambda_{i} f^{i}\right)(x)=\Sigma \lambda_{i} f^{\prime}(x)$.
} 
second reason for needing calculus and topology is the integration of Social Choice into the general body of Economic Theory. This should help avoid the rather specialized, often obscure nature of the results obtained in this area by means of the single technique of discrete combinatorial analysis. An example is provided by the results relating social choice and the theory of markets with non convexity in Chichilnisky $(1990 \mathrm{~b})$. These two reasons militate against Baigent and Huang's arguments. Unless they wish to keep Social Choice separate from the rest of economic theory, and unless they wish to avoid the flexibility introduced by the use of several different mathematical techniques, they should favour the topological approach. I think that their intention is to integrate the results obtained with the topological approach into the main body of Social Choice by providing interpretations which are easier to understand with the more familiar combinatorial techniques. By all means we should employ different techniques and test their usefulness against each other. It is the most efficient way to advance our knowledge in a difficult area: we need all the tools we can get. I do not advocate the superiority of the topological approach. I advocate the superiority of a flexible attitude towards techniques and approaches.

Finally, let me emphasize the urgency with which I feel Social Choice should be integrated into the general body of economic theory, particularly with respect to the analysis of markets. Democracy is studied and tested in Social Choice. Markets are studied and tested in General Equilibrium Theory. The relationship between these two fundamental concepts can only be studied in depth once we integrate the two fields, as discussed in Chichilnisky $(1990 \mathrm{a}, \mathrm{b})$. For this we need a common language, and common techniques.

\section{References}

Baigent $N$ (1984) A reformulation of Chichilnisky's impossibility theorem. Econ Lett 16: $23-25$

Baigent N (1985) Anonymity and continuous social choice. J Math Econ 13:1-4

Baigent N (1987) Preference proximity and anonymous social choice. Q J Econ 102:162-169

Baigent N (1989) Some further remarks on preference proximily. Q J Econ 104: 191-193

Baigent N, Huang P (1990) Topological social choice: reply to LeBreton and Uriarte. Soc Choice Welfare $7: 141-146$

Black D (1948) On the rational of group decision making. I Polit Econ 56: 23-34

Chichilnisky G (1979) On fixed point theorems and social choice paradoxes. Econ Lett 3: 347-351

Chichilnisky G (1980) Social choice and the topology of spaces of prelerences. Adv Math 37: $165-176$

Chichilnisky G (1982) Social aggregation rules and continuity. Q J Econ 96:337-352

Chichilnisky G (1983) Social choice and game theory: recent results with a topological approach. Pattanaik PK, Salles M (eds) Social Choice and Welfare, chap 6. North-Holland, Amsterdam, pp 79-102

Chichilnisky G (1990a) On the mathematical foundation of political cconomy. Political Fconomy Iecture, Harvard University, March 22, 1990. Contr Polit Econ 9: 25-31

Chichilnisky $\mathrm{G}$ (1990b) General equilibrium and social choice with increasing returns. Ann Oper Res 23: 289-297

Chichilnisky G, Heal GM (1983) Necessary and sufficient conditions for a resolution of the social choice paradox. J Econ Theory $31: 68-87$ 
Chichilnisky G, Heal GM (1984) Patterns of power. I Publ Econ, pp 333-349

Debreu G (1972) Smooth preferences. Econometrica 40: $6003-615$

LeBreton M, Uriarte JR (1990a) On the robustness of the impossibility result in the topological approach to social choice. Soc Choice Welfare $7: 131-140$

LeBreton M, Uriarte JR (1990b) Topological social choice: a rejoinder. Soc Choice Welfare 7: $147-148$

Spanier E (1966) Algebraic topology. MeGraw-Hill, New York 